\title{
$S U_{L}(2) \times U_{Y}(1)$-invariant description of the bilepton contribution to the $W W V$ vertex in the minimal 331 model
}

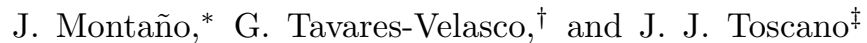 \\ Facultad de Ciencias Físico Matemáticas, Benemérita Universidad \\ Autónoma de Puebla, Apartado Postal 1152, Puebla, Pue., México \\ F. Ramírez-Zavaletas \\ Departamento de Física, CINVESTAV, Apartado Postal 14-740, 07000, México D. F., México
}

(Dated: July 17, 2019)

\begin{abstract}
We study the one-loop sensitivity of the $W W V(V=\gamma, Z)$ vertex to the new massive gauge bosons predicted by the minimal $S U_{L}(3) \times U_{X}(1)$ model, which have unusual couplings to the standard model (SM) gauge bosons. A gauge-fixing procedure covariant under the $S U_{L}(2) \times U_{Y}(1)$ group was introduced for these new gauge bosons (dubbed bileptons) in order to generate gauge-invariant Green functions. The similarities between this procedure and the nonconventional quantization scheme of the background field method are discussed. It is found that, for relatively light bileptons, with a mass ranging from $2 m_{W}$ to $6 m_{W}$, the radiative corrections to the form factors associated with the $W W V$ vertex can be of the same order of magnitude than the SM one. In the case of heavier bileptons, their contribution is smaller by about one and two orders of magnitude than their SM counterpart.
\end{abstract}

PACS numbers: 12.60.Cn,14.70.Hp,13.40.Gp

\section{INTRODUCTION}

The $W W V(V=\gamma, Z)$ one-loop structure has been considerably studied in the literature not just because it may constitute a mechanism through which physics beyond the Fermi scale may show up, but also due to some theoretical issues concerning its dependence on the gauge-fixing scheme. It turns out that the conventional gaugefixing procedures give rise to ill-behaved off-shell Green functions that may display inadequate properties such as a nontrivial dependence on the gauge-fixing parameter, an increase larger than the one observed in physical amplitudes at high energies, and the appearance of unphysical thresholds. The on-shell Green functions can represent physical amplitudes as they are independent on the gauge-fixing procedure, such as occurs with the static electromagnetic properties of the $W$ boson [1, 2, 3], but gauge independence is lost if at least one external particle becomes virtual. Although off-shell Green functions are generally gauge dependent, the $S$-matrix elements to which they contribute must be gauge independent. This is the case of the off-shell $W W V$ vertex, which is just a piece of some physical process such as the $e^{+} e^{-} \rightarrow W^{+} W^{-}$and $\gamma \gamma \rightarrow W^{+} W^{-}$reactions. Nonetheless, it would be interesting if one was able to study the sensitivity to radiative corrections of the $W W V$ coupling, and other SM couplings as well, without invoking some particular $S$-matrix element.

The concepts of gauge invariance and gauge independence are two essential ingredients of gauge systems, though the former is not necessarily present at the quantum level. While gauge invariance plays a central role when defining the classical action of the system, once the latter is quantized one must invariably invoke an appropriate gaugefixing procedure to define a nondegenerate action, which means that gauge invariance is to be broken explicitly. The resultant action is not gauge invariant, though it is invariant under BRST symmetry [4]. As a consequence, the Green functions derived from this action cannot satisfy simple (QED-like) Ward identities, but they do satisfy more elaborate Slavnov-Taylor identities that are dictated by BRST symmetry. Also, Green functions contain much unphysical information that is removed provided a physical observable is considered. Contrary to Green functions, which are highly dependent on the gauge-fixing procedure, physical amplitudes have no such dependence, thereby being gauge independent. There are thus some subtle mechanisms that conspire to produce nontrivial cancelations between the Green functions defining a physical observable. It is clear that a nonconventional quantization scheme

\footnotetext{
*E-mail montano@fcfm.buap.mx

${ }^{\dagger}$ E-mail gtv@fcfm.buap.mx

‡E-mail jtoscano@fcfm.buap.mx

§E-mail rzf@fis.cinvestav.mx
} 
must be applied in order to generate gauge-invariant Green functions, which in turn can be obtained from a gaugeinvariant quantum action $\Gamma$. In this respect, the background-field-method (BFM) [5] is meant to construct manifest gauge-invariant quantum actions from which well-behaved Green functions satisfying simple Ward identities can be derived. This method, implemented at the level of generating functionals, relies on the decomposition of the gauge fields into two parts: ${ }^{1}$ the quantum field $A_{\mu}^{a}$ and the background (classical) field $\hat{A}_{\mu}^{a}$, i.e. $A_{\mu}^{a} \rightarrow A_{\mu}^{a}+\hat{A}_{\mu}^{a}$. In the generating functional only the quantum fields are integrated out, whereas the background fields are treated as sources. This means that only the quantum fields can circulate inside the loops. This method allows one to introduce a gaugefixing procedure for the quantum fields without spoiling the gauge invariance of the quantum action with respect to the classical fields. Although it is necessary to define a gauge-fixing procedure for both the quantum and the classical fields in order to define $S$-matrix elements, only a gauge-fixing scheme for the quantum gauge fields is required to define general off-shell Green functions. The quantum action is invariant under ordinary gauge transformations of the classical fields, while the quantum fields transform as the adjoint representation of the group in consideration. In other words, the so constructed action $S[A+\hat{A}]$ is degenerate with respect to the background fields but nondegenerate with respect to the quantum fields. The Green functions derived from the quantum action $\Gamma[\hat{A}]$ are gauge invariant in the sense that they satisfy simple Ward identities, but it is worth stressing that they are still dependent on the gauge parameter $\xi_{Q}$ that characterizes the gauge-fixing scheme used for the quantum fields, and so there is no gauge independence. The BFM has proved useful in many applications [7], simplifying both technically and conceptually the calculation of radiative corrections.

As already mentioned, Green functions arising from a conventional quantum action (a BRST-invariant but gaugenoninvariant one) contain a lot of unphysical information that is removed once they are inserted into some physical observable. Some of this unwanted information can be removed at the level of the generating functional through the BFM formalism, which allows one to construct a gauge-invariant quantum action from which gauge-invariant Green functions can be obtained. Although the resultant Green functions satisfy simple Ward identities, they are not gauge independent. Thus far there is still no known mechanism yielding both gauge-invariant and gauge-independent Green functions directly from the generating functional, although there is already a diagrammatic method meant for this purpose, the so-called pinch technique (PT) [8]. This method relies on constructing well-behaved Green functions by combining some individual contributions from self-energies, vertex, and box diagrams, which usually appear in physical processes. In general, the Feynman rules used in this diagrammatic approach are derived from a conventional effective action, though those derived from the BFM have also been used for a deeper study of the method selfconsistence [9]. Although the PT was first introduced for the study of pure Yang-Mills theories at the one-loop level [8, 9, 10], it has already been applied to theories with spontaneous symmetry breaking (SSB) [1]], including the study of self-energies [12] and trilinear vertices [13] involving the electroweak gauge bosons. A complete calculation of the one-loop contribution to the $W W V$ vertex from the electroweak bosons was presented in Refs. [14, 15]: it was intended to show that the vertex functions satisfy a simple Ward identity, which establishes a relationship between this vertex and the $W$ self-energy. More specifically, Ref. [14] discusses the gauge independence of the form factors associated with the $W W \gamma$ vertex for off-shell photon and on-shell $W$ bosons. Afterwards, an important connection between the PT and the BFM was established [16] at the one-loop level by showing that the Green functions calculated via the BFM Feynman rules coincide with those obtained through the PT for the specific value $\xi_{Q}=1$. More recently, the PT was extended to the two-loop level in the context of both the Yang-Mills [17] and the electroweak sectors [18], and the one-loop connection to the BFM was established too. A step toward a nondiagrammatic formulation of the PT via the powerful Batalin-Vilkovisky quantization method [19| was presented in Ref. [20]. This framework was used to generalize the PT at any order of perturbation theory [21], and it was meant to show that the link between the PT Green functions and those obtained via the BFM along with the Feynman-t'Hooft gauge remains at all orders of perturbation theory [21, 22]. The reason for such a link remains a puzzle, though it is worth noting that the Feynmant'Hooft gauge yields no unphysical thresholds. Establishing such a connection at any order of perturbation theory is very important for practical purposes because one can simply use the BFM Feynman-t'Hooft gauge (BFMFG) to calculate gauge-independent off-shell amplitudes, which happens to be much less cumbersome than the use of the PT.

Although in conventional quantization schemes the quantum action of the theory is not gauge invariant, it is still possible to introduce gauge invariance with respect to a subgroup of such a theory. This scheme is particulary useful when the quantum fluctuations of the gauge fields associated with this subgroup are deemed negligible. For instance, it would be interesting to assess the virtual effects of the heavy physics lying beyond the Fermi scale on the SM Green functions in a $S U_{L}(2) \times U_{Y}(1)$-covariant manner, in which case it is only necessary to introduce a quantization scheme for the heavy fields since the SM fields would only appear as external legs. This is indeed the philosophy behind the effective Lagrangian approach widely used in the context of the electroweak theory, where it is assumed

\footnotetext{
${ }^{1}$ Indeed, every bosonic field must be decomposed into a quantum and a classic part [6].
} 
that the new physics effects must respect the $S U_{L}(2) \times U_{Y}(1)$ symmetry. In a specific theory beyond the SM, a $S U_{L}(2) \times U_{Y}(1)$-invariant effective Lagrangian can be constructed by introducing a $S U_{L}(2) \times U_{Y}(1)$-covariant gaugefixing procedure for the heavy gauge bosons in order to integrate them out in the generating functional. In analogy with the BFM, the gauge-fixing procedure for the heavy gauge fields must involve the $S U_{L}(2) \times U_{Y}(1)$-covariant derivative given in the representation in which the heavy fields transform under this group. This is the reason why such gauges, which were first introduced by Fujikawa in the context of the SM [23], are called nonlinear or covariant gauges. In this case, the $W$ propagator is defined in a covariant way under the electromagnetic $U_{e}(1)$ group, so the vertex functions associated with the $W W \gamma$ interaction and the $W$ self-energy satisfy a simple Ward identity. The most general renormalizable structure of this gauge-fixing procedure has been discussed from the BRST-symmetry standpoint in 24], and a discussion about the difficulties on implementing the Faddeev-Popov method (FPM) has been presented too 25]. This gauge-fixing procedure has proved a valuable tool in radiative corrections as it simplifies considerably the loop calculations [26]. The method has also been used to quantize Yang-Mills theories without SSB 27]. We will show below that, within some specific models, it is possible to use a nonlinear gauge to parametrize in an $S U_{L}(2) \times U_{Y}(1)$-invariant way the impact of new physics on the SM Green functions. In particular, we will show that it is possible to use this class of gauges to estimate the one-loop effects of new heavy gauge bosons on the $W W V$ vertex.

We are interested in the sensitivity of the $W W V$ vertex to the new heavy gauge bosons predicted by the so-called minimal 331 model [28, 29], which is based on the $S U_{C}(3) \times S U_{L}(3) \times U_{X}(1)$ gauge group. Apart from predicting signals of new physics at the TeV scale, this model introduces unique features that have been the focus of great interest recently [30] such as a possible approach to the solution of the family replication problem. In this model, the lepton spectrum is the same as the $\mathrm{SM}$ one, but it is accommodated in $S U_{L}(3)$ antitriplets; the quark sector is also arranged in the fundamental representation of this group, which requires the introduction of three new quarks. In order to endow all the particles with mass, a Higgs sector composed by three triplets and one sextet of $S U_{L}(3)$ is required, though only one of the triplets is needed to break down $\left.S U_{L}(3) \times U_{X}\right)(1)$ into $S U_{L}(2) \times U_{Y}(1)$ at the new physics scale $u>v$. In the first stage of SSB, there emerge singly and doubly charged gauge bosons in a doublet of the $S U_{L}(2)$ group, as well as a new neutral boson $Z^{\prime}$. The new charged gauge bosons were dubbed bileptons because they carry two units of lepton number. The three exotic quarks and a CP even Higgs boson do not couple to the $W$ gauge boson since they emerge as singlets of $S U_{L}(2)$, and get their mass at the $u$ scale. Thus, at this scale, the $W W \gamma$ and $W W Z$ vertices can only receive contributions from the bileptons. The fact that the $S U_{L}(2)$ group is totally embedded in $S U_{L}(3)$ gives rise to unusual couplings between the bileptons and the SM gauge fields, which arise via the electroweak covariant derivative since the bileptons transform as the fundamental representation of $S U_{L}(2)$. It turns out that these couplings do not involve any mixing angle and are similar both in strength and Lorentz structure to those couplings existing between the SM gauge bosons themselves, as opposed to the gauge bosons appearing in other SM extensions. Our main goal is to estimate, in a $S U_{L}(2) \times U_{Y}(1)$-invariant way, the sensitivity of the $W W V$ vertex to the bileptons. To this end, we introduce a $S U_{L}(2) \times U_{Y}(1)$-covariant gauge-fixing procedure for the bileptons, which leads to an invariant quantum action. We will show below that the resulting $W W \gamma$ and $W W Z$ Green functions are gauge invariant and satisfy simple Ward identities. Another feature worthwhile to emphasize is that the FPM fails when it is attempted to be used in conjunction with this class of gauges: the resultant theory is not renormalizable 24]. Instead of using this method, we will present a discussion based on BRST symmetry [4], which is a powerful formalism adequate not only to quantize Yang-Mills theories with broader gauge-fixing procedures, as the nonlinear ones, but also to quantize more general gauge systems. As we will see below, our quantization scheme incorporates the main ingredient of the BFM, namely, the gauge invariance of the quantum action, which turns loop calculations into a somewhat simple task.

The rest of the paper has been organized as follows. In Sec. II a brief description of the minimal 331 model is presented. Particular emphasis is given to the Yang-Mills sector. In Sec. III] a $S U_{L}(2) \times U_{Y}(1)$-covariant gauge-fixing procedure for the bileptons is presented along with a discussion on the advantages of using the BRST formalism instead of the FPM. Sec. IV is devoted to present the one-loop amplitudes for the $W W \gamma$ and $W W Z$ vertices, whereas in Secs. $\nabla$ and $\nabla \mathbf{D}$ we discuss our results and present the conclusions.

\section{THE MINIMAL 331 MODEL}

The $S U_{C}(3) \times S U_{L}(3) \times U_{X}(1)$ model has been discussed to some extent in the literature 3 , 30, 31. We will only focus on those features that are relevant for the present discussion. In particular, we will concentrate on the first stage of SSB, when the 331 group is broken down into the SM group. The complete Higgs sector is comprised by three triplets and one sextet of $S U_{L}(3)$, but only the following triplet is necessary to break $S U_{L}(3) \times U_{X}(1)$ into 
$S U_{L}(2) \times U_{Y}(1)$

$$
\Phi=\left(\begin{array}{c}
\Phi_{Y} \\
\phi^{0}
\end{array}\right): \quad(1,3,1)
$$

where $\Phi_{Y}$ is a doublet of $S U_{L}(2) \times U_{Y}(1)$ with hypercharge 3 .

In the fundamental representation of $S U_{L}(3) \times U_{X}(1)$, the covariant derivative can be written as

$$
\mathcal{D}_{\mu}=\partial_{\mu}-i g \frac{\lambda^{a}}{2} A_{\mu}^{a}-i g_{X} X \frac{\lambda^{9}}{2} X_{\mu}, \quad(a=1, \cdots, 8),
$$

where $\lambda^{a}(a=1, \cdots, 8)$ are the Gell-Mann matrices and $\lambda^{9}=\sqrt{2 / 3} \operatorname{diag}(1,1,1)$. The generators are normalized as $\operatorname{Tr} \lambda^{a} \lambda^{b}=2 \delta^{a b}$. When $\Phi$ develops a vacuum expectation value, $\Phi_{0}^{\dagger}=(0,0, u / \sqrt{2})$, the exotic quarks, one physical neutral scalar, and the new gauge bosons acquire masses, whereas the remaining scalar multiplets give mass to the SM particles. The first stage of SSB is accomplished by $\Phi_{0}$ according to the following scheme: six generators are broken $\left(\lambda^{b} \Phi_{0} \neq 0\right.$ for $\left.b=4, \cdots, 9\right)$ and the remaining ones leave invariant the vacuum $\left(\lambda^{a} \Phi_{0}=0\right.$ for $\left.a=1,2,3\right)$. Notice that $\sqrt{3}\left(\lambda^{8}+\sqrt{2} X \lambda^{9}\right) \Phi_{0}=0$, so the hypercharge can be identified with the following linear combination of broken generators: $Y=\sqrt{3}\left(\lambda^{8}+\sqrt{2} X \lambda^{9}\right)$. At this stage of SSB there appear one single charged bilepton and one doubly charged one defined by

$$
\begin{aligned}
Y_{\mu}^{++} & =\frac{1}{\sqrt{2}}\left(A_{\mu}^{4}-i A_{\mu}^{5}\right), \\
Y_{\mu}^{+} & =\frac{1}{\sqrt{2}}\left(A_{\mu}^{6}-i A_{\mu}^{7}\right),
\end{aligned}
$$

which have the following mass

$$
m_{Y^{++}}=m_{Y^{+}}=m_{Y}=\frac{g u}{2} .
$$

According to the quantum number assignment, these fields accommodate in one doublet of $S U_{L}(2) \times U_{Y}(1)$ with hypercharge 3:

$$
Y_{\mu}=\left(\begin{array}{c}
Y_{\mu}^{++} \\
Y_{\mu}^{+}
\end{array}\right)
$$

As far as the scalar triplet is concerned, the two components of the $S U_{L}(2) \times U_{Y}(1)$ doublet with hypercharge $3, \Phi_{Y}$, coincide with the pseudo-Goldstone bosons associated with the bilepton doublet:

$$
\Phi_{Y}=\left(\begin{array}{c}
G_{Y}^{++} \\
G_{Y}^{+}
\end{array}\right) .
$$

Finally, the third component of the triplet contains the physical Higgs boson and the pseudo-Goldstone boson associated with $Z^{\prime}$ :

$$
\phi^{0}=\frac{1}{\sqrt{2}}\left(u+H^{\prime 0}+i G_{Z^{\prime}}\right) .
$$

The bilepton masses receive new contributions at the Fermi scale, when $S U_{L}(2) \times U_{Y}(1)$ is broken into $U_{e}(1)$, which yields an upper bound on the splitting between the square bilepton masses:

$$
\left|m_{Y^{++}}^{2}-m_{Y^{+}}^{2}\right| \leq m_{W}^{2} .
$$

Therefore, $m_{Y^{++}}$and $m_{Y^{+}}$cannot not be very different: one of them cannot become arbitrarily large while the other one remains fixed. In fact, the bilepton masses become nearly degenerate when they are much larger than $m_{W}$. In addition, the theoretical constraint $4 s_{W}^{2} \leq 1$ obtained from matching the gauge couplings constants at the $S U_{L}(3) \times U_{X}(1)$ breaking scale yields an upper bound on the bilepton masses of the order of $1 \mathrm{TeV}$ [29, 32]. Therefore, our estimate for the Green functions associated with the $W W V$ vertex at the $u$ scale would not become spoiled by 
the new contributions arising at the $v$ scale. As far as the remaining gauge bosons are concerned, the gauge fields $A_{\mu}^{8}$ and $X_{\mu}$ mix to produce a massive field $Z_{\mu}^{\prime}$, and a massless gauge boson $B_{\mu}[31$. The latter is associated with the $U_{Y}(1)$ group. At the $u$ scale, the $Z^{\prime}$ field does not couple with the $W$ boson, though it can couple to a $W$ boson pair at the Fermi scale via $Z^{\prime}-Z$ mixing 31].

Therefore, although five massive gauge bosons emerge at the $u$ scale, along with three exotic quarks and one Higgs boson, only the bileptons couple to the $W$ gauge boson. These interactions are dictated by the $S U_{L}(2) \times$ $U_{Y}(1)$ symmetry, and emerge entirely from the Yang-Mills sector associated with the $S U_{L}(3) \times U_{X}(1)$ group. The corresponding Lagrangian is composed of the following three $S U_{L}(2) \times U_{Y}(1)$-invariant pieces [3] :

$$
\mathcal{L}_{Y M}=-\frac{1}{4} F_{\mu \nu}^{a} F_{a}^{\mu \nu}-\frac{1}{4} X_{\mu \nu} X^{\mu \nu}=\mathcal{L}_{S M}+\mathcal{L}_{S M N P}+\mathcal{L}_{N P}
$$

where $\mathcal{L}_{S M}$ is the electroweak Yang-Mills Lagrangian:

$$
\mathcal{L}_{S M}=-\frac{1}{4} W_{\mu \nu}^{i} W_{i}^{\mu \nu}-\frac{1}{4} B_{\mu \nu} B^{\mu \nu}
$$

where we have made the association $A_{\mu}^{a} \rightarrow W_{\mu}^{i}$, for $a=1,2,3 . \mathcal{L}_{S M N P}$ encompasses the interactions between the SM gauge bosons and the new ones, it can be written as

$$
\begin{aligned}
\mathcal{L}_{S M N P}= & -\frac{1}{2}\left(D_{\mu} Y_{\nu}-D_{\nu} Y_{\mu}\right)^{\dagger}\left(D^{\mu} Y^{\nu}-D^{\nu} Y^{\mu}\right)-Y^{\dagger \mu}\left(i g \mathbf{W}_{\mu \nu}+i g^{\prime} \mathbf{B}_{\mu \nu}\right) Y^{\nu} \\
& -\frac{i g \sqrt{3} \sqrt{1-4 s_{W}^{2}}}{2 c_{W}} Z_{\mu}^{\prime}\left[Y_{\nu}^{\dagger}\left(D^{\mu} Y^{\nu}-D^{\nu} Y^{\mu}\right)-\left(D^{\mu} Y^{\nu}-D^{\nu} Y^{\mu}\right)^{\dagger} Y_{\nu}\right]
\end{aligned}
$$

where we have introduced the definitions $\mathbf{W}_{\mu \nu}=\tau^{i} W_{\mu}^{i} / 2$ and $\mathbf{B}_{\mu \nu}=Y B_{\mu \nu} / 2$. In addition, $D_{\mu}=\partial_{\mu}-i g \mathbf{W}_{\mu}-i g^{\prime} \mathbf{B}_{\mu}$ stands for the covariant derivative associated with the electroweak group. The first two terms of this Lagrangian induce a diversity of couplings between the SM gauge bosons and the bileptons. Finally, the term $\mathcal{L}_{N P}$ induces the interactions between the $Z^{\prime}$ boson and the bileptons:

$$
\begin{aligned}
\mathcal{L}_{N P}= & -\frac{1}{4} Z_{\mu \nu}^{\prime} Z^{\prime \mu \nu}-\frac{i g \sqrt{3} \sqrt{1-4 s_{W}^{2}}}{2 c_{W}} Z_{\mu \nu}^{\prime} Y^{\dagger \mu} Y^{\nu}-\frac{3 g^{2}\left(1-4 s_{W}^{2}\right)}{4 c_{W}^{2}} Z_{\mu}^{\prime} Y_{\nu}^{\dagger}\left(Z^{\prime \mu} Y^{\nu}-Z^{\prime \nu} Y^{\mu}\right) \\
& +\frac{g^{2}}{2}\left(Y_{\mu}^{\dagger} \frac{\tau^{i}}{2} Y_{\nu}\right)\left(Y^{\dagger \mu} \frac{\tau^{i}}{2} Y^{\nu}-Y^{\dagger \nu} \frac{\tau^{i}}{2} Y^{\mu}\right)+\frac{3 g^{2}}{4}\left(Y_{\mu}^{\dagger} Y_{\nu}\right)\left(Y^{\dagger \mu} Y^{\nu}-Y^{\dagger \nu} Y^{\mu}\right) .
\end{aligned}
$$

Note that each term in the last two Lagrangians is separately invariant under the electroweak group.

As evident from above, the one-loop level contributions to the $W W \gamma$ and $W W Z$ vertices arise only from the first two terms appearing in $\mathcal{L}_{S M N P}$. A gauge-fixing procedure covariant under the $S U_{L}(2) \times U_{Y}(1)$ group will be introduced below for the bilepton sector.

\section{GAUGE-FIXING PROCEDURE AND FEYNMAN RULES}

It has already been mentioned that the FPM fails to quantize Yang-Mills theories possessing more general supplementary conditions than the linear ones. This stems from the fact that the FPM leads to an action which is bilinear in the ghost and antighost fields since they arise essentially from the integral representation of a determinant. This is not however the most general situation that can arise since an action including quartic ghost interactions at the tree level is still consistent with BRST symmetry and the power counting criterion of renormalization theory. It turns out that the FPM does succeed when applied to linear gauges because quartic ghost interactions cannot arise from loop effects due to antighost translation invariance, ${ }^{2}$ which stems from the fact that the antighost fields appear only through their derivatives. However, this symmetry is lost in the case of nonlinear gauges since the gauge-fixing functions depend on bilinear terms of gauge fields. These terms are responsible for the presence of ultraviolet-divergent quartic-ghost

\footnotetext{
${ }^{2}$ Invariance under the transformation $\bar{C}^{a} \rightarrow \bar{C}^{a}+c^{a}$, with $c^{a}$ arbitrary constant parameters.
} 
interactions at one-loop level. This means that renormalizability becomes ruined when the FPM is attempted to be used in the context of nonlinear gauges. It is thus convenient to discard the FPM and building up instead the most general action consistent with BRST symmetry and renormalization theory. BRST symmetry arises naturally from the field-antifield formalism [19], which has proved a powerful tool in quantizing gauge systems. In the case of Yang-Mills systems, the gauge-fixed BRST action has a simple structure [19]:

$$
S_{B R S T}=S_{331}+\delta \Psi
$$

where $S_{331}$ is the gauge-invariant classical action, $\delta$ is the BRST operator, and $\Psi$ is the fermion action. $S_{B R S T}$ would be nondegenerate if a gauge-fixing procedure for all the gauge fields of the model was introduced. Since we are only interested in the virtual effects of the bileptons, a gauge-fixing procedure for these fields is only necessary. Furthermore, we use a gauge-fixing procedure covariant under the $S U_{L}(2) \times U_{Y}(1)$ group because we are interested in preserving such a symmetry. The resultant $S_{B R S T}$ action is nondegenerate with respect to the bilepton fields, ${ }^{3}$ but degenerate with respect to the electroweak fields. As a consequence, a $S U_{L}(2) \times U_{Y}(1)$-invariant quantum action can be constructed out of which gauge-invariant Green functions, $<0\left|W_{\mu}^{+}\left(x_{1}\right) W_{\nu}^{-}\left(x_{2}\right) A_{\lambda}\left(x_{3}\right)\right| 0>$ and $<0\left|W_{\mu}^{+}\left(x_{1}\right) W_{\nu}^{-}\left(x_{2}\right) Z_{\lambda}\left(x_{3}\right)\right| 0>$, satisfying simple Ward identities, can be derived. More specifically, we introduce a fermion action defined as follows:

$$
\Psi=\int d^{4} x\left[\bar{C}^{\bar{a}}\left(f^{\bar{a}}+\frac{\xi}{2} B^{\bar{a}}+f^{\bar{a} b c} \bar{C}^{b} C^{c}\right)\right], \quad \bar{a}=4,5,6,7 ; \quad b, c=1, \cdots 8,
$$

where $f^{\bar{a}}, \bar{C}^{\bar{a}}$, and $B^{\bar{a}}$ are the gauge-fixing functions, the antighost fields, and the auxiliary scalar fields associated with the $A_{\mu}^{\bar{a}}$ gauge fields, respectively. In addition, $C^{a}$ are the ghost fields associated with the $A_{\mu}^{a}$ fields, $f^{a b c}$ are the $S U_{L}(3)$ structure constants, and $\xi$ is the gauge parameter. Note that the $f^{\bar{a} b c} \bar{C}^{b} C^{c}$ term cannot arise from the FPM, though its presence is necessary to obtain renormalizability when the gauge-fixing functions are nonlinear. Using the usual BRST transformations, we obtain for the $\Psi$ variation

$$
\delta \Psi=\int d^{4} x\left[\frac{\xi}{2} B^{\bar{a}} B^{\bar{a}}+\left(f^{\bar{a}}+2 f^{\bar{a} b c} \bar{C}^{b} C^{c}\right) B^{\bar{a}}-\bar{C}^{\bar{a}}\left(\delta f^{\bar{a}}\right)-\frac{1}{2} f^{\bar{a} b c} f^{c d e} \bar{C}^{\bar{a}} \bar{C}^{b} C^{d} C^{e}\right] .
$$

On the other hand, since the auxiliary fields $B^{\bar{a}}$ appear quadratically, they can be integrated out in the generating functional. Since the coefficients of the quadratic terms do not depend on the fields, their integration is equivalent to applying the equations of motion to the gauge-fixed BRST action. Once these steps are done, we obtain an action defined by the following $S U_{L}(2) \times U_{Y}(1)$-invariant Lagrangian

$$
\mathcal{L}_{B R S T}=\mathcal{L}_{331}+\mathcal{L}_{G F}+\mathcal{L}_{F P}
$$

where $\mathcal{L}_{331}$ is the gauge invariant Lagrangian of the 331 model, whereas $\mathcal{L}_{G F}$ and $\mathcal{L}_{F P}$ arise from the action $\delta \Psi$. The former is the gauge-fixing term, which can be written as

$$
\mathcal{L}_{G F}=-\frac{1}{2 \xi} f^{\bar{a}} f^{\bar{a}}
$$

and $\mathcal{L}_{F P}$ represents the ghost sector:

$$
\mathcal{L}_{F P}=-\bar{C}^{\bar{a}}\left(\delta f^{\bar{a}}\right)-\frac{2}{\xi} f^{\bar{a} b c} f^{\bar{a}} \bar{C}^{b} C^{c}-\frac{1}{2} f^{\bar{a} b c} f^{c d e} \bar{C}^{\bar{a}} \bar{C}^{b} C^{d} C^{e} .
$$

While the first term in this Lagrangian does arise when the FPM is used, the remaining ones are new and must be preserved if a nonlinear function $f^{\bar{a}}$ is introduced. If a linear gauge is used, these terms can be removed after invoking antighost-translation invariance.

We are now ready to introduce the most general $S U_{L}(2) \times U_{Y}(1)$-covariant $f^{\bar{a}}$ functions, and we will take advantage of the fact that every coupling involving at least one pseudo-Goldstone boson and every coupling with gauge freedom can be modified leaving unaltered the $S$ matrix. The most general renormalizable gauge-fixing functions consistent with this symmetry can be written as

$$
f^{\bar{a}}=\left(\delta^{\bar{a} b} \partial_{\mu}-g f^{\bar{a} b i} A_{\mu}^{i}\right) A^{\mu b}-\frac{\xi g}{\sqrt{3}} f^{\bar{a} b 8} \Phi^{\dagger} \lambda^{b} \Phi, \quad \bar{a}=4,5,6,7 ; \quad i=1,2,3,8 .
$$

\footnotetext{
${ }^{3}$ If necessary, a gauge-fixing procedure for the new $Z$ boson can be introduced without affecting the $S U_{L}(2) \times U_{Y}(1)$-invariance of $S_{B R S T}$.
} 
Notice that these gauge-fixing functions are nonlinear in both the vector and the scalar sectors. To be fully aware of the covariant structure of these gauge-fixing functions, it is convenient to express them in terms of the mass eigenstates fields. Using the definitions

$$
\begin{aligned}
f_{Y}^{++} & =\frac{1}{\sqrt{2}}\left(f^{4}-i f^{5}\right), \\
f_{Y}^{+} & =\frac{1}{\sqrt{2}}\left(f^{6}-i f^{7}\right),
\end{aligned}
$$

we can write

$$
f_{Y}=\left(\begin{array}{c}
f_{Y}^{++} \\
f_{Y}^{+}
\end{array}\right)=\left(D_{\mu}-\frac{i g \sqrt{3} \sqrt{1-4 s_{W}^{2}}}{2 c_{W}} Z_{\mu}^{\prime}\right) Y^{\mu}-\frac{i g \xi}{\sqrt{2}} \phi^{0 *} \Phi_{Y},
$$

where $D_{\mu}$ is the $S U_{L}(2) \times U_{Y}(1)$-covariant derivative given in the doublet representation. From this expression, it is evident that $f_{Y}$ transforms as $Y_{\mu}$ or $\Phi_{Y}$, i.e. as a doublet of $S U_{L}(2) \times U_{Y}(1)$ with hypercharge 3 . As a consequence, the gauge-fixing term $\mathcal{L}_{G F}$ is manifestly invariant under this group. As will become evident below, it is convenient to decompose the Lagrangian $\mathcal{L}_{G F}$ into three gauge-invariant terms:

$$
\mathcal{L}_{G F}=\mathcal{L}_{G F 1}+\mathcal{L}_{G F 2}+\mathcal{L}_{G F 3},
$$

where

$$
\begin{aligned}
\mathcal{L}_{G F 1}= & -\frac{1}{\xi}\left(D_{\mu} Y^{\mu}\right)^{\dagger}\left(D_{\nu} Y^{\nu}\right)-\frac{\xi g^{2}}{2}\left(\phi^{0 *} \phi^{0}\right)\left(\Phi_{Y}^{\dagger} \Phi_{Y}\right), \\
\mathcal{L}_{G F 2}= & \frac{i g}{\sqrt{2}}\left[\phi^{0 *}\left(D_{\mu} Y^{\mu}\right)^{\dagger} \Phi_{Y}-\phi^{0} \Phi_{Y}^{\dagger}\left(D_{\mu} Y^{\mu}\right)\right] \\
\mathcal{L}_{G F 3}= & \frac{i g \sqrt{3} \sqrt{1-4 s_{W}^{2}}}{2 c_{W} \xi} Z_{\mu}^{\prime}\left[\left(D_{\nu} Y^{\nu}\right)^{\dagger} Y^{\mu}-Y^{\mu \dagger}\left(D_{\nu} Y^{\nu}\right)\right] \\
& -\frac{g^{2} \sqrt{3} \sqrt{1-4 s_{W}^{2}}}{2 \sqrt{2} c_{W}} Z_{\mu}^{\prime}\left(\phi^{0 *} Y^{\mu \dagger} \Phi_{Y}+\phi^{0} \Phi_{Y}^{\dagger} Y^{\mu}\right) \\
& -\frac{3 g^{2}\left(1-4 s_{W}^{2}\right)}{4 c_{W}^{2} \xi} Z_{\mu}^{\prime} Z_{\nu}^{\prime} Y^{\mu \dagger} Y^{\nu} .
\end{aligned}
$$

We would like to discuss the dynamics of $\mathcal{L}_{G F 1}$ and $\mathcal{L}_{G F 2}$. The first term appearing in $\mathcal{L}_{G F 1}$, which is invariant under the electroweak group, not only allows to define the bilepton propagators but also modifies nontrivially the couplings between the bileptons and the electroweak gauge bosons appearing in the $\mathcal{L}_{N P S M}$ Lagrangian. When the two Lagrangians are combined, they lead to trilinear and quartic vertices:

$$
\begin{gathered}
\mathcal{L}_{W Y Y}=i e_{W}\left\{W^{+\mu}\left(Y_{\mu \nu}^{--} Y^{+\nu}-Y_{\mu \nu}^{+} Y^{--\nu}\right)-W_{\mu \nu}^{+} Y^{--\mu} Y^{+\nu}-W^{-\mu}\left(Y_{\mu \nu}^{++} Y^{-\nu}-Y_{\mu \nu}^{-} Y^{++\nu}\right)+W_{\mu \nu}^{-} Y^{++\mu} Y^{-\nu}\right. \\
\left.+\frac{1}{\xi}\left[W^{+\mu}\left(Y_{\mu}^{+} \partial_{\nu} Y^{--\nu}-Y_{\mu}^{--} \partial_{\nu} Y^{+\nu}\right)-W^{-\mu}\left(Y_{\mu}^{-} \partial_{\nu} Y^{++\nu}-Y_{\mu}^{++} \partial_{\nu} Y^{-\nu}\right)\right]\right\} \\
\mathcal{L}_{V Y Y}=i e_{V}\left\{Q_{Y^{+}}^{V}\left[V^{\mu}\left(Y_{\mu \nu}^{-} Y^{+\nu}-Y_{\mu \nu}^{+} Y^{-\nu}\right)-V_{\mu \nu} Y^{-\mu} Y^{+\nu}+\frac{1}{\xi} V^{\mu}\left(Y_{\mu}^{+} \partial_{\nu} Y^{-\nu}-Y_{\mu}^{-} \partial_{\nu} Y^{+\nu}\right)\right]+\right. \\
Q_{Y^{++}}^{V}\left[V^{\mu}\left(Y_{\mu \nu}^{--} Y^{++\nu}-Y_{\mu \nu}^{++} Y^{--\nu}\right)-V_{\mu \nu} Y^{--\mu} Y^{++\nu}+\frac{1}{\xi} V^{\mu}\left(Y_{\mu}^{++} \partial_{\nu} Y^{--\nu}-Y_{\mu}^{--} \partial_{\nu} Y^{++\nu}\right)\right], \\
\mathcal{L}_{W W Y Y}=-e_{W}^{2}\left\{W_{\mu}^{-} W^{+\mu}\left(Y_{\nu}^{-} Y^{+\nu}+Y_{\nu}^{--} Y^{++\nu}\right)\right. \\
-W_{\mu}^{-} W_{\nu}^{+}\left(2 Y^{+\mu} Y^{-\nu}-Y^{-\mu} Y^{+\nu}+2 Y^{--\mu} Y^{++\nu}-Y^{--\nu} Y^{++\mu}\right) \\
\left.+\frac{1}{\xi} W_{\mu}^{-} W_{\nu}^{+}\left(Y^{-\mu} Y^{+\nu}+Y^{--\nu} Y^{++\mu}\right)\right\}
\end{gathered}
$$




$$
\begin{aligned}
\mathcal{L}_{V W Y Y}= & -e_{W} e_{V} V^{\mu}\left\{\left(Q_{Y^{+}}^{V}+Q_{Y^{++}}^{V}\right)\left[Y^{--\nu}\left(W_{\mu}^{+} Y_{\nu}^{+}-W_{\nu}^{+} Y_{\mu}^{+}\right)+Y^{++\nu}\left(W_{\mu}^{-} Y_{\nu}^{-}-W_{\nu}^{-} Y_{\mu}^{-}\right)\right]\right. \\
& +\left(Q_{Y^{++}}^{V}-2 Q_{Y^{+}}^{V}\right)\left[W^{+\nu}\left(Y_{\mu}^{--} Y_{\nu}^{+}-Y_{\nu}^{--} Y_{\mu}^{+}\right)+W^{-\nu}\left(Y_{\mu}^{++} Y_{\nu}^{-}-Y_{\nu}^{++} Y_{\mu}^{-}\right)\right] \\
& \left.+\frac{1}{\xi}\left[Q_{Y^{+}}^{V}\left(Y_{\nu}^{--} W^{+\nu} Y_{\mu}^{+}+Y_{\nu}^{++} W^{-\nu} Y_{\mu}^{-}\right)+Q_{Y^{++}}^{V}\left(Y_{\mu}^{--} W_{\nu}^{+} Y^{+\nu}+Y_{\mu}^{++} W_{\nu}^{-} Y^{-\nu}\right)\right]\right\},
\end{aligned}
$$

where $e_{V}=e, Q_{Y^{+}}^{V}=1$, and $Q_{Y^{++}}^{V}=2$ for $V=\gamma$, whereas $e_{V}=g /\left(2 c_{W}\right), Q_{Y^{+}}^{V}=-\left(1+2 s_{W}^{2}\right)$, and $Q_{Y^{++}}^{V}=1-4 s_{W}^{2}$,

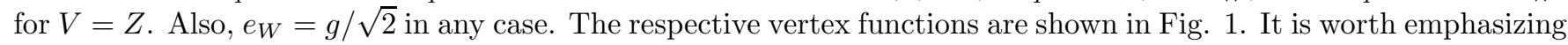
that, as required by $S U_{L}(2) \times U_{Y}(1)$ symmetry, the vertex functions associated with the trilinear couplings $W Y Y$ and $V Y Y$ share the same Lorentz structure:

$$
\Gamma_{\alpha \mu \nu}\left(k, k_{1}, k_{2}\right)=\left(k_{2}-k_{1}\right)_{\alpha} g_{\mu \nu}+\left(k-k_{2}-\frac{1}{\xi} k_{1}\right)_{\mu} g_{\alpha \nu}-\left(k-k_{1}-\frac{1}{\xi} k_{2}\right)_{\nu} g_{\alpha \mu} .
$$

It is not hard to show that it satisfies the following simple Ward identity

$$
k^{\alpha} \Gamma_{\alpha \mu \nu}\left(k, k_{1}, k_{2}\right)=\Pi_{\mu \nu}^{Y^{\dagger} Y^{\dagger}}\left(k_{2}\right)-\Pi_{\mu \nu}^{Y Y}\left(k_{1}\right),
$$

where $\Pi_{\mu \nu}^{Y \dagger Y \dagger}\left(k_{2}\right)$ and $\Pi_{\mu \nu}^{Y Y}\left(k_{1}\right)$ are two-point vertex functions given by

$$
\Pi_{\mu \nu}^{Y Y}(k)=\left(-k^{2}+m_{Y}^{2}\right) g_{\mu \nu}-\left(\frac{1}{\xi}-1\right) k_{\mu} k_{\nu}
$$

As far as the quartic vertices are concerned, they are characterized by the following vertex functions:

$$
\begin{gathered}
\Gamma_{\alpha \beta \mu \nu}^{W W Y Y}=g_{\alpha \beta} g_{\mu \nu}-2 g_{\alpha \nu} g_{\beta \mu}+\left(1+\frac{1}{\xi}\right) g_{\alpha \mu} g_{\beta \nu}, \\
\Gamma_{\alpha \beta \mu \nu}^{V W Y Y}=\left(Q_{Y+}^{V}+Q_{Y++}^{V}\right)\left(g_{\alpha \beta} g_{\mu \nu}-g_{\alpha \nu} g_{\beta \mu}\right)+3 \delta_{V Z}\left(g_{\alpha \mu} g_{\beta \nu}-g_{\alpha \nu} g_{\beta \mu}\right)+\frac{1}{\xi}\left(Q_{Y+}^{V} g_{\alpha \nu} g_{\beta \mu}+Q_{Y++}^{V} g_{\alpha \mu} g_{\beta \nu}\right) .
\end{gathered}
$$

On the other hand, the scalar part of $\mathcal{L}_{G F 1}$ allows one to define unphysical masses for the pseudo-Goldstone bosons associated with the bileptons, and it also modifies some unphysical couplings of the Higgs potential.

It is worthwhile to discuss the dynamics of the $\mathcal{L}_{G F 2}$ Lagrangian. This term has a strong impact on the Higgs kinetic-energy sector associated with the $\Phi$ triplet as it helps to remove some unphysical vertices. Note that the latter can be decomposed into three $S U_{L}(2) \times U_{Y}(1)$-invariant pieces:

$$
\left(\mathcal{D}_{\mu} \Phi\right)^{\dagger}\left(\mathcal{D}^{\mu} \Phi\right)=\mathcal{L}_{K \Phi 1}+\mathcal{L}_{K \Phi 2}+\mathcal{L}_{K \Phi 3},
$$

where

$$
\mathcal{L}_{K \Phi 1}=\left(D_{\mu} \Phi_{Y}\right)^{\dagger}\left(D^{\mu} \Phi_{Y}\right)+\partial_{\mu} \phi^{0 *} \partial^{\mu} \phi^{0}+\frac{g^{2}}{2}\left[\phi^{0 *} \phi^{0} Y_{\mu}^{\dagger} Y^{\mu}+\left(\Phi_{Y}^{\dagger} Y_{\mu}\right)\left(Y^{\mu \dagger} \Phi_{Y}\right)\right]
$$

and

$$
\mathcal{L}_{K \Phi 2}=i e_{W}\left[\phi^{0 *} Y_{\mu}^{\dagger}\left(D^{\mu} \Phi_{Y}\right)+\Phi_{Y}^{\dagger} Y_{\mu} \partial^{\mu} \phi^{0}-\text { H.c. }\right] .
$$

The $\mathcal{L}_{K \Phi 3}$ Lagrangian is not relevant for the present discussion as it is only composed by the interactions involving the $Z^{\prime}$ boson, so we refrain from presenting it here. The Lagrangian $\mathcal{L}_{K \Phi 1}$ gives rise to the interactions between the pseudo-Goldstone bosons and the electroweak gauge bosons. These interactions, which are dictated by the electroweak group, contribute to the $W W \gamma$ and $W W Z$ couplings at the one-loop level, so the corresponding Feynman rules are shown in Fig. [2 On the other hand, the $\mathcal{L}_{K \Phi 2}$ term is responsible for the appearance of the bilinear terms $Y_{\mu}^{ \pm \pm} G_{Y}^{\mp \mp}$ and $Y_{\mu}^{ \pm} G_{Y}^{\mp}$, as well as the unphysical trilinear and quartic couplings $Y_{\mu}^{ \pm \pm} W^{\mp} G_{Y}^{\mp}, Y_{\mu}^{ \pm} W^{ \pm} G_{Y}^{\mp \mp}, H^{\prime} Y_{\mu}^{ \pm \pm} W^{\mp} G_{Y}^{\mp}$, and $H^{\prime} Y_{\mu}^{ \pm} W^{ \pm} G_{Y}^{\mp \mp}$. When the $\mathcal{L}_{G F 2}$ Lagrangian is taken into account, all these couplings vanish. In fact, after adding up these two terms, we obtain

$$
\mathcal{L}_{K \Phi 2}+\mathcal{L}_{G F 2}=i e_{W}\left[\phi^{0 *} \partial_{\mu}\left(Y^{\mu \dagger} \Phi_{Y}\right)+\Phi_{Y}^{\dagger} Y_{\mu} \partial^{\mu} \phi^{0}-\text { H.c. }\right]
$$




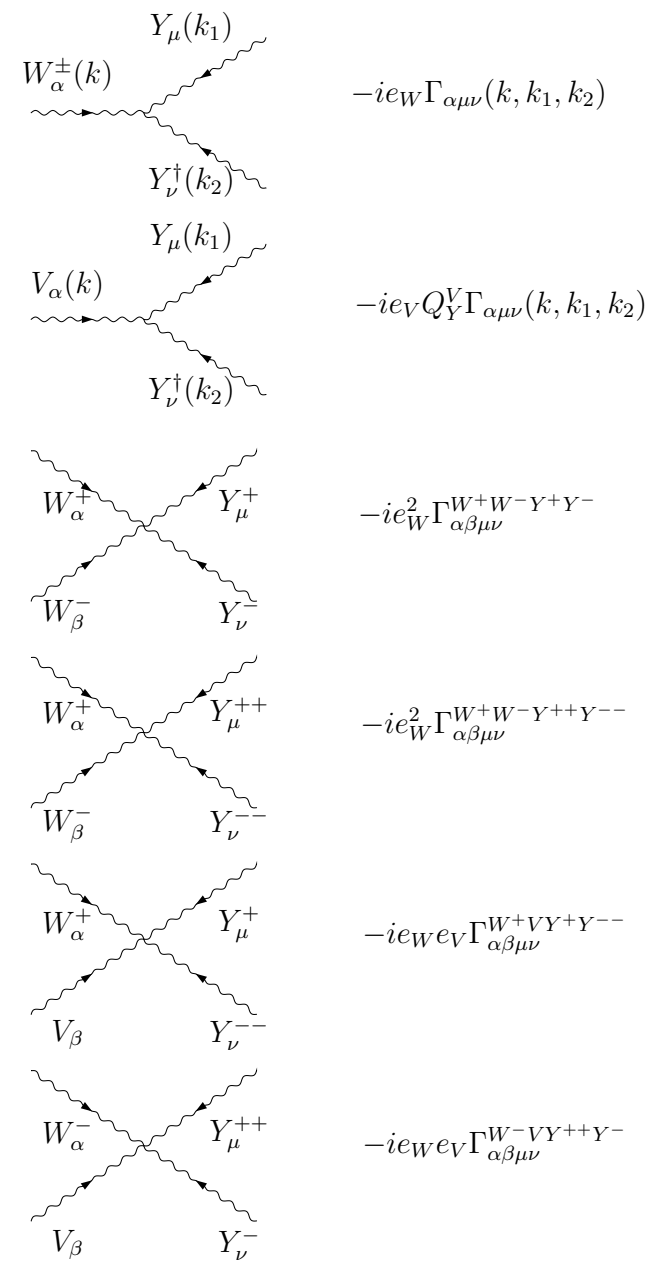

FIG. 1: Feynman rules for the trilinear and quartic vertices involving the bileptons and SM gauge fields in the $S U_{L}(2) \times U_{Y}(1)$ covariant $R_{\xi}$-gauge.

where some surface terms were ignored. Needless to say that the absence of these unphysical vertices renders great simplicity for some loop calculations.

As far as the ghost sector is concerned, the following definitions for the ghost fields

$$
\begin{aligned}
C_{Y}^{ \pm \pm} & =\frac{1}{\sqrt{2}}\left(C^{4} \mp i C^{5}\right), \\
C_{Y}^{ \pm} & =\frac{1}{\sqrt{2}}\left(C^{6} \mp i C^{7}\right),
\end{aligned}
$$

and similar expressions for the antighost fields, allow us to express the corresponding Lagrangian as follows:

$$
\begin{aligned}
\mathcal{L}_{F P}= & \left(D_{\mu} C_{Y}\right)^{\dagger}\left(D^{\mu} \bar{C}_{Y}\right)+\frac{g^{2}}{4}\left[\left(Y_{\mu}^{\dagger} \sigma^{i} Y^{\mu}\right)\left(C_{Y}^{\dagger} \sigma^{i} \bar{C}_{Y}\right)+3\left(Y_{\mu}^{\dagger} Y^{\mu}\right)\left(C_{Y}^{\dagger} \bar{C}_{Y}\right)-4\left(Y_{\mu}^{\dagger} C_{Y}\right)\left(Y^{\mu \dagger} \bar{C}_{Y}\right)\right] \\
& +\frac{i g}{\sqrt{2}} Y_{\mu}^{\dagger} M_{C} D^{\mu} \bar{C}_{Y}+\frac{i g}{2} Y_{\mu}^{\dagger} \mathcal{M}_{C} \bar{C}_{Y}-\frac{\xi g}{2}\left[\phi^{0 *} \phi^{0} C_{Y}^{\dagger} \bar{C}_{Y}+\phi^{0} \Phi_{Y}^{\dagger} M_{C} \bar{C}_{Y}-\left(C_{Y}^{\dagger} \Phi_{Y}\right)\left(\Phi_{Y}^{\dagger} \bar{C}_{Y}\right)\right] \\
& +\frac{i \sqrt{2}}{\xi}\left[\left(\bar{M}_{C} C_{Y}+M_{C} \bar{C}_{Y}\right)^{\dagger}\left(D_{\mu} Y^{\mu}\right)-\left(D_{\mu} Y^{\mu}\right)\left(\bar{M}_{C} C_{Y}+M_{C} \bar{C}_{Y}\right)\right] \\
& -g\left[\Phi_{Y}^{\dagger}\left(\bar{M}_{C} C_{Y}+M_{C} \bar{C}_{Y}\right) \phi^{0}+\phi^{0 *}\left(\bar{M}_{C} C_{Y}+M_{C} \bar{C}_{Y}\right)^{\dagger} \Phi_{Y}\right]+\text { H.c. } \\
& -\frac{1}{2} f^{\bar{a} b c} f^{c d e} \bar{C}^{\bar{a}} \bar{C}^{b} C^{d} C^{e},
\end{aligned}
$$


where

$$
C_{Y}=\left(\begin{array}{c}
C_{Y}^{++} \\
C_{Y}^{+}
\end{array}\right) \quad \bar{C}_{Y}=\left(\begin{array}{c}
\bar{C}_{Y}^{++} \\
\bar{C}_{Y}^{+}
\end{array}\right)
$$

They have the same quantum numbers that $Y_{\mu}$ and $\Phi_{Y}$. In addition,

$$
\begin{gathered}
M_{C}=\left(\begin{array}{cc}
\frac{1}{\sqrt{2}}\left(C^{3}+\sqrt{3} C^{8}\right) & \frac{1}{\sqrt{2}}\left(C^{1}-i C^{2}\right) \\
\frac{1}{\sqrt{2}}\left(C^{1}+i C^{2}\right) & -\frac{1}{\sqrt{2}}\left(C^{3}-\sqrt{3} C^{8}\right)
\end{array}\right), \\
\mathcal{M}_{C}=\left(\begin{array}{cc}
\left(\mathcal{D}_{\mu}^{3 i}+\sqrt{3} \mathcal{D}_{\mu}^{8 i}\right) C^{i} & \left(\mathcal{D}_{\mu}^{1 i}-i \mathcal{D}_{\mu}^{21}\right) C^{i} \\
\left(\mathcal{D}_{\mu}^{1 i}+i \mathcal{D}_{\mu}^{2 i}\right) C^{i} & -\left(\mathcal{D}_{\mu}^{3 i}-\sqrt{3} \mathcal{D}_{\mu}^{8 i}\right) C^{i}
\end{array}\right),
\end{gathered}
$$

where $i=1,2,3,8$ and $\mathcal{D}_{\mu}^{i j}=\delta^{i j} \partial_{\mu}-g f^{i j a} A_{\mu}^{a}$ stands for the covariant derivative given in the adjoint representation of $S U_{L}(3)$. The $\bar{M}_{C}$ matrix is obtained from $M_{C}$ after replacing the ghost fields by antighost fields. Under the electroweak group, $M_{C}$ transforms as $M_{C} \rightarrow U M_{C} U^{\dagger}$, with $U \in S U_{L}(2) \times U_{Y}(1)$. A similar transformation holds for $\bar{M}_{C}$ and $\mathcal{M}_{C}$. As a consequence, $\mathcal{L}_{F P}$ is invariant under the $S U_{L}(2) \times U_{Y}(1)$ group.

Both the pseudo-Goldstone boson and the ghost sectors contribute to the vertex $W W V$ via trilinear and quartic couplings. As shown in Fig. 22 the Feynman rules arising from each sector are identical because each sector is $S U_{L}(2) \times U_{Y}(1)$ invariant by its own. As a consequence, the trilinear vertices $W S^{\dagger} S$ and $V S^{\dagger} S$ satisfy simple Ward identities:

$$
k^{\alpha} \Gamma_{\alpha}^{V S^{\dagger} S}=\Pi^{S^{\dagger} S^{\dagger}}\left(k_{2}\right)-\Pi^{S S}\left(k_{1}\right),
$$

where $\Gamma_{\alpha}^{V S^{\dagger} S}=\left(k_{1}-k_{2}\right)_{\alpha}, S$ stands for a commutative (pseudo-Goldstone boson) or anticommutative (ghost) charged scalar, and $\Pi^{S S}\left(k_{i}\right)$ stands for the two-point vertex functions $\Pi\left(k_{i}\right)=k_{i}^{2}-\xi m_{Y}^{2}$.

\section{THE ONE-LOOP $W W V$ VERTEX}

The prospect of the NLC and CLIC [33], have triggered the interest in the $e^{+} e^{-} \rightarrow W^{+} W^{-}$reaction, and motivated by this we will focus on the $W W V$ vertex with the two $W \mathrm{~s}$ on-shell and $V$ off-shell. Even in this case there is no reason to expect a gauge-invariant amplitude arising from a conventional quantization scheme. We will show below that this is indeed the case. Retaining just the transverse degrees of freedom of $V$, the vertex function for the $W W V$ coupling can be written as [1, 34]:

$$
\Gamma_{\alpha \beta \mu}^{V}=-i g_{V}\left\{A\left[2 p_{\mu} g_{\alpha \beta}+4\left(q_{\beta} g_{\alpha \mu}-q_{\alpha} g_{\beta \mu}\right)\right]+2 \Delta \kappa_{V}\left(q_{\beta} g_{\alpha \mu}-q_{\alpha} g_{\beta \mu}\right)+\frac{4 \Delta Q_{V}}{m_{W}^{2}}\left(p_{\mu} q_{\alpha} q_{\beta}-\frac{1}{2} q^{2} p_{\mu} g_{\alpha \beta}\right)\right\},
$$

where

$$
g_{V}=e_{V}\left(Q_{Y^{++}}^{V}-Q_{Y^{+}}^{V}\right)= \begin{cases}g s_{W}, & V=\gamma \\ g c_{W}, & V=Z\end{cases}
$$

We have dropped the CP-odd terms since they do not arise at the one-loop level in the minimal 331 model. Our notation and conventions are depicted in Fig. 3. In the SM, the tree level values are $A=1, \Delta \kappa=0$, and $\Delta Q=0$. In the case of the on-shell $W W \gamma$ vertex, $\Delta \kappa_{\gamma}$ and $\Delta Q_{\gamma}$ are related to the $W$ magnetic dipole moment $\mu_{W}$ and the electric quadrupole moment $Q_{W}$ :

$$
\begin{aligned}
\mu_{W} & =\frac{e}{2 m_{W}}\left(2+\Delta \kappa_{\gamma}\right), \\
Q_{W} & =-\frac{e}{m_{W}^{2}}\left(1+\Delta \kappa_{\gamma}+\Delta Q_{\gamma}\right) .
\end{aligned}
$$

As already noted, we are interested in the impact of the bileptons on the $\Delta \kappa_{V}$ and $\Delta Q_{V}$ form factors, which characterize the radiative corrections to the $W W V$ vertex. Our results will only be an estimate since we are only considering the new physics effects at the $u$ scale, but our approach has the advantage of invariance under the $S U_{L}(2) \times U_{Y}(1)$ group, which greatly simplifies the calculations. At the $u$ scale, only the bileptons couple to the $W$ boson since they arise as a doublet of the electroweak group. We already presented the Feynman rules given in a 

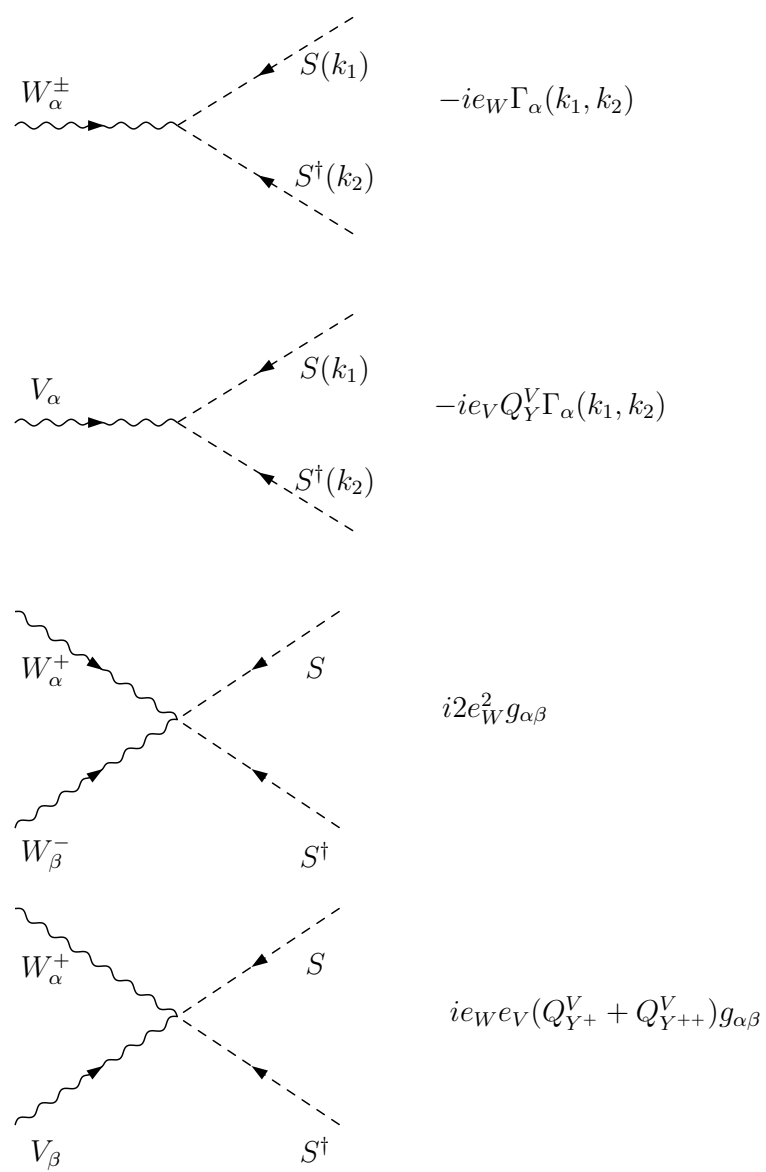

FIG. 2: Feynman rules for the trilinear and quartic vertices involving SM gauge fields and scalar unphysical particles (pseudoGoldstone bosons and ghosts) in the $S U_{L}(2) \times U_{Y}(1)$-covariant $R_{\xi}$-gauge. In this gauge, the $W$ and $V$ couplings to pseudoGoldstone bosons and ghosts coincide.

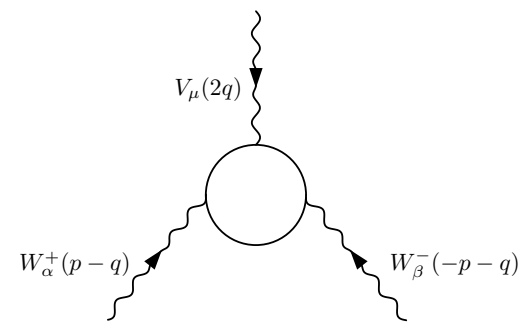

FIG. 3: The trilinear $W W V$ vertex. The large circle denotes loop contributions and the arrows denote the flow of momenta.

$R_{\xi}$-gauge scheme and their $S U_{L}(2) \times U_{Y}(1)$-covariant nature was displayed via simple Ward identities. The one-loop amplitude of the $W W V$ vertex will also be gauge invariant, though gauge dependent, which also occurs when the $\mathrm{BFM}$ is applied. In other words, gauge-invariant quantum actions render gauge-invariant but not gauge-independent Green functions. However, motivated by the link between the BFMFG and the PT, we will present our results in the Feynman-t'Hooft gauge.

The generic Feynman diagrams contributing to the $W W V$ vertex are shown in Fig. 4 It turns out that the bileptons contribute through all these diagrams, but the only nonvanishing contribution of scalar particles arises from the triangle graphs. In addition, owing to the separate $S U_{L}(2) \times U_{Y}(1)$ invariance of the ghost and scalar sectors, the ghost-antighost contribution is exactly minus twice the one coming from the pseudo-Goldstone bosons. Taking into account all these facts, the total amplitude can be written as

$$
\Gamma_{\alpha \beta \mu}^{V}=-g_{V} I_{\alpha \beta \mu}
$$




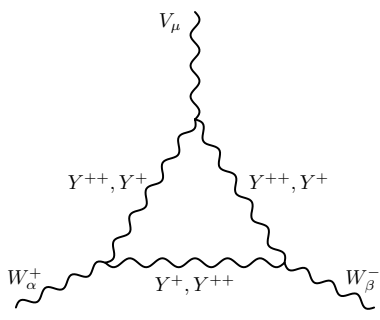

(a)

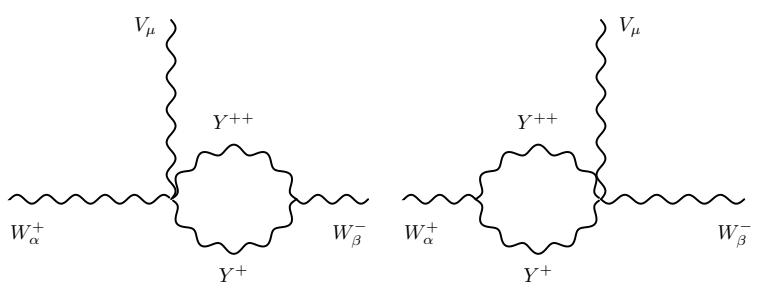

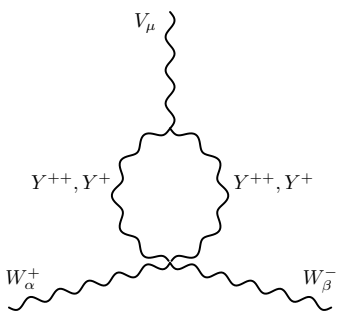

(b)

(d)

FIG. 4: Feynman diagrams for the $W W V$ vertex in the $S U_{L}(2) \times U_{Y}(1)$-covariant gauge. The pseudo-Goldstone bosons and ghosts contribute through an identical set of diagrams, but only the triangle ones give a nonvanishing contribution to the form factors $\Delta \kappa_{V}$ and $\Delta Q_{V}$.

with $I_{\alpha \beta \mu}$ the loop amplitude, which is the same for both $W W \gamma$ and $W W Z$ vertices. It is worth mentioning that the associated Green functions differ only by the factor $g_{V}$, just as occurs at the tree-level. This means that $S U_{L}(2) \times U_{Y}(1)$ invariance is preserved at the one-loop level. Thus the loop amplitude $I_{\alpha \beta \mu}$ for on-shell $W$ bosons must satisfy the simple Ward identity

$$
q^{\mu} I_{\alpha \beta \mu}=0
$$

which can be verified once the loop integrals are solved explicitly. The explicit calculation shows that the $\Delta \kappa_{V}$ and $\Delta Q_{V}$ form factors are given by

$$
\begin{aligned}
\Delta \kappa_{V} & =\frac{6 a}{\left(4 x_{W}-1\right)^{3}}\left\{x_{W}\left(4 x_{W}-1\right)\left(8 x_{W}+3\right)-6 x_{W}\left[x_{W}\left(1+x_{W}\right)+3 x_{Y}\left(1-4 x_{W}\right)\right] Q^{2} C_{0}\right. \\
& \left.+4 x_{Y}\left(4 x_{W}-1\right)^{2}\left[B_{0}(3)-B_{0}(1)\right]+\left[26 x_{W}^{2}+32 x_{Y} x_{W}\left(1-4 x_{W}\right)+x_{W}\right]\left[B_{0}(1)-B_{0}(2)\right]\right\}, \\
\Delta Q_{V}= & \frac{12 a}{\left(4 x_{W}-1\right)^{3}}\left\{6 x_{W}\left[2 x_{W}\left(2 x_{W}^{3}-2 x_{W}^{2}\left(1+4 x_{Y}\right)+x_{W}\left(1+6 x_{Y}\right)-3 x_{Y}\right)+x_{Y}\right] Q^{2} C_{0}\right. \\
& +4 x_{W}\left(x_{W}\left(6 x_{W}^{2}-5 x_{W}+8 x_{Y}-1\right)-2 x_{Y}\right)\left[B_{0}(1)-B_{0}(2)\right]+4 x_{W} x_{Y}\left(4 x_{W}-1\right)^{2}\left[B_{0}(2)-B_{0}(3)\right] \\
& \left.-x_{W}\left(4 x_{W}-1\right)\left(1+2 x_{W}\left(6 x_{W}-1\right)\right)\right\},
\end{aligned}
$$

where we have introduced the definitions $Q=2 q, a=g^{2} / 96 \pi^{2}, x_{W}=m_{W}^{2} / Q^{2}$, and $x_{Y}=m_{Y}^{2} / Q^{2}$. $B_{0}(i)$ and $C_{0}$ stand for the following Passarino-Veltman scalar functions: $B_{0}(1)=B_{0}\left(m_{W}^{2}, m_{Y}^{2}, m_{Y}^{2}\right), B_{0}(2)=B_{0}\left(Q^{2}, m_{Y}^{2}, m_{Y}^{2}\right)$, $B_{0}(3)=B_{0}\left(0, m_{Y}^{2}, m_{Y}^{2}\right)$, and $C_{0}=C_{0}\left(Q^{2}, m_{W}^{2}, m_{W}^{2}, m_{Y}^{2}, m_{Y}^{2}, m_{Y}^{2}\right)$.

\section{DISCUSSION}

The $e^{+} e^{-} \rightarrow W^{+} W^{-}$reaction will play an essential role in future researches at $e^{+} e^{-}$colliders: it will provide relevant information for our knowledge of the SM such as a more precise determination of the $W$ mass and its 
width decays, and it will also open up the possibility for detecting new physics effects via the distinctive $s$-channel contribution from the $W W V$ vertex. In the SM, the radiative corrections to the $e^{+} e^{-} \rightarrow W^{+} W^{-}$process have been widely studied both for on-shell [35] and off-shell $W$ gauge bosons [36]. New physics effects have also been studied in a model-independent manner using the effective Lagrangian approach [37]. Beyond the SM, the reaction $e^{+} e^{-} \rightarrow W^{+} W^{-}$has been analyzed in technicolor theories [38] and supersymmetric models [39].

As for the radiative corrections to the $W W V$ vertex, they have received considerably attention. In the SM, the one-loop amplitudes were calculated using the conventional quantization scheme along with the Feynman-t'Hoof gauge [40]. As emphasized in that work, the resultant amplitudes are not gauge invariant, which is evident from the presence of infrared divergences and the bad high-energy behavior of the $\Delta \kappa_{V}$ form factor [40]. In contrast, it was found that $\Delta Q$ is well-behaved. Shortly afterwards, these vertices were revisited by Papavassiliou and Philippides [4] in a gauge-invariant way via the PT. The form factor $\Delta \kappa_{V}$ obtained by these authors disagrees from that presented in [40], though there is agreement for $\Delta Q_{V}$. It was found that the radiative corrections to $\Delta \kappa_{V}$ are of the order of $\alpha / \pi$, whereas $\Delta Q_{V}$ is about one order of magnitude below. For instance, $\Delta \kappa_{\gamma}$ goes from $10^{-3}$ for $Q=200 \mathrm{GeV}$ to $10^{-4}$ for $Q=1000 \mathrm{GeV}$ [4], whereas $\Delta Q_{\gamma}$ ranges from $10^{-4}$ to $6 \times 10^{-5}$ in the same energy range [4, 41]. As far as experimental measurements are concerned, the constraint $\left|\Delta \kappa_{V}\right|,\left|\Delta Q_{V}\right| \lesssim 1 / 2$ was obtained from CERN 42 and Fermilab data 43]. It is expected that this constraint is substantially improved at the CERN large hadron collider (LHC). Even more, it has been argued that a deviation at the $10^{-3}$ level might be measured at NLCs. As a consequence, only $\Delta \kappa_{V}$ would be at the reach of NLCs, though the appearance of new physics effects may improve this situation. This is not the case however for one of the more popular SM extensions, namely, supersymmetry, which yields similar or smaller contributions than the SM ones [44].

We turn now to our results. The $\Delta \kappa_{V}$ and $\Delta Q_{V}$ form factors depend on $Q^{2}, m_{Y}$, and $m_{W}$. As for $Q^{2}$, it can take both positive (time-like) and negative (space-like) values. However, motivated by the prospect of NLCs, the form factors will be evaluated for $Q>100 \mathrm{GeV}$. It is also evident that for relative low energies, where the SM contribution is dominant, those contributions of very heavy bileptons will be highly suppressed. However, when $Q$ and $m_{Y}$ are of the same order, it is expected that the new physics contributions become more relevant. One interesting feature of the minimal 331 model is the constraint $s_{W}^{2}<1 / 4$ obtained from theoretical arguments, which in turn translates into $m_{Y} \lesssim 1.5 \mathrm{TeV}$ in the minimal 331 model [32], though it can be relaxed by introducing a more complex Higgs sector. We will consider this upper constraint for the bilepton mass. On the other hand, the most stringent lower bound $m_{Y}>850 \mathrm{GeV}$ [45] arises from muonium-antimuonium conversion. It has been argued however that this bound can be evaded in a more general context since it relies on very restrictive assumptions [46]. Other strong limit, $m_{Y}>750$ $\mathrm{GeV}$, arises from fermion pair production and lepton flavor violating decays [47], and the bound $m_{Y \pm}>440 \mathrm{GeV}$, valid only for the singly charged bilepton mass, was derived from limits on the muon decay width [48]. We would like to stress that all these bounds depend on several assumptions, and in principle there still remains the possibility of lighter bileptons. Although it is quite unlikely the existence of a relatively light bilepton, as way of illustration, we will concentrate on the range $2 m_{W}<m_{Y}<12 m_{W}$. Also, although the contribution of a bilepton is expected to become more significant when its mass is of the same order of magnitude than that of $Q$, we will present results in the range $100 \mathrm{GeV}<Q<1000 \mathrm{GeV}$.

The $\Delta \kappa_{V}$ and $\Delta Q$ form factors are shown in Figs. 5 and 6 for $m_{Y}=2 m_{W}, 4 m_{W}, 8 m_{W}$, and $12 m_{W}$. We have included the values that arise above the threshold $Q \geq 2 m_{Y}$ only by completeness, as in such a situation it would be more appropriate to study the direct production of bilepton pairs rather than their virtual effects. From those Figures we can see that the $\Delta \kappa_{V}$ and $\Delta Q_{V}$ signs are reversed, a situation also observed in the SM model. For larger values of $m_{Y}$ the form factors increase with the energy, although both of them approach asymptotically to zero for very large $|Q|$ after reaching an extremum. This situation is similar to what is observed in the SM after the PT is implemented 14, 15]. From Fig. [5] we can see that $\Delta \kappa_{V}$ ranges between $10^{-4}$ and $10^{-5}$ for a relatively light bilepton with a mass in the range $2 m_{W}<m_{Y}<8 m_{W}$. These values are of the same order of magnitude than the SM contribution. As far as $\Delta Q_{V}$ is concerned, it ranges from $10^{-4}$ to $10^{-5}$ in the same $m_{Y}$ range, which means that this form factor has essentially the same behavior than the SM contribution. Also, we can see that a more heavy bilepton, with a mass in the range $8 m_{W}<m_{Y}<12 m_{W}$, yields both $\Delta \kappa_{V}$ and $\Delta Q_{V}$ at the $10^{-6}$ level, and they increase smoothly when the energy increases. This means that for very heavy bileptons, $\Delta \kappa_{V}$ and $\Delta Q_{V}$ are one order of magnitude smaller than the respective SM radiative correction. Notice also that the inequality $\left|\Delta Q_{V}\right|>\left|\Delta \kappa_{V}\right|$ always holds, which is opposite to what is observed in the SM. This apparent contradiction stems from the fact that, in our case, the new physics effects are of decoupling nature: the form factors vanish in the limit of a very large mass $m_{Y}$. Indeed, $\Delta Q_{V}$ is always of decoupling nature since it arises from a nonrenormalizable dimension-six operator. In contrast, $\Delta \kappa_{V}$ can be sensitive to nondecoupling effects since it is associated with a renormalizable Lorentz structure of dimension four. Its nondecoupling nature is well known from the SM [1, 41] and some of its extensions [2, 44].

Finally, we would like to comment our results within the context of the effective Lagrangian framework. In this scheme, the electroweak Lagrangian is extended with nonrenormalizable operators of dimension higher than four respecting the $S U_{L}(2) \times U_{Y}(1)$ symmetry. In particular, anomalous contributions to the $\Delta \kappa_{V}$ and $\Delta Q_{V}$ form factors 

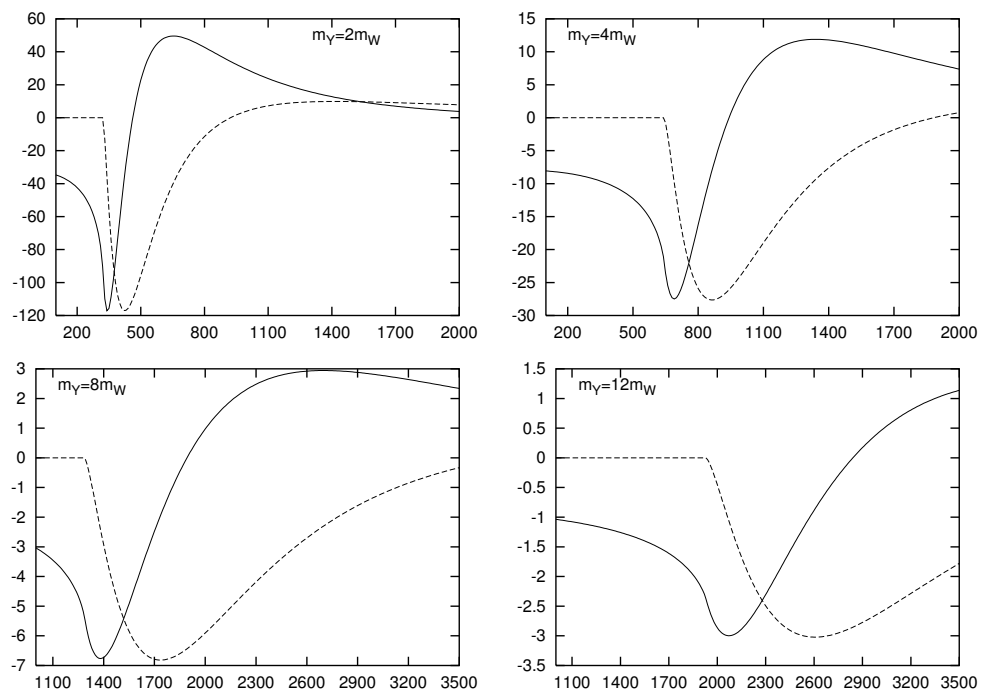

FIG. 5: The $\Delta \kappa_{V}$ form factor, in units of $10^{-6}$, as a function of the center-of-mass energy $|Q|=\sqrt{s}$, in units of GeV, for several values of $m_{Y}$. The solid line is for the real part of $\Delta \kappa_{V}$ and the dashed line for its imaginary part.
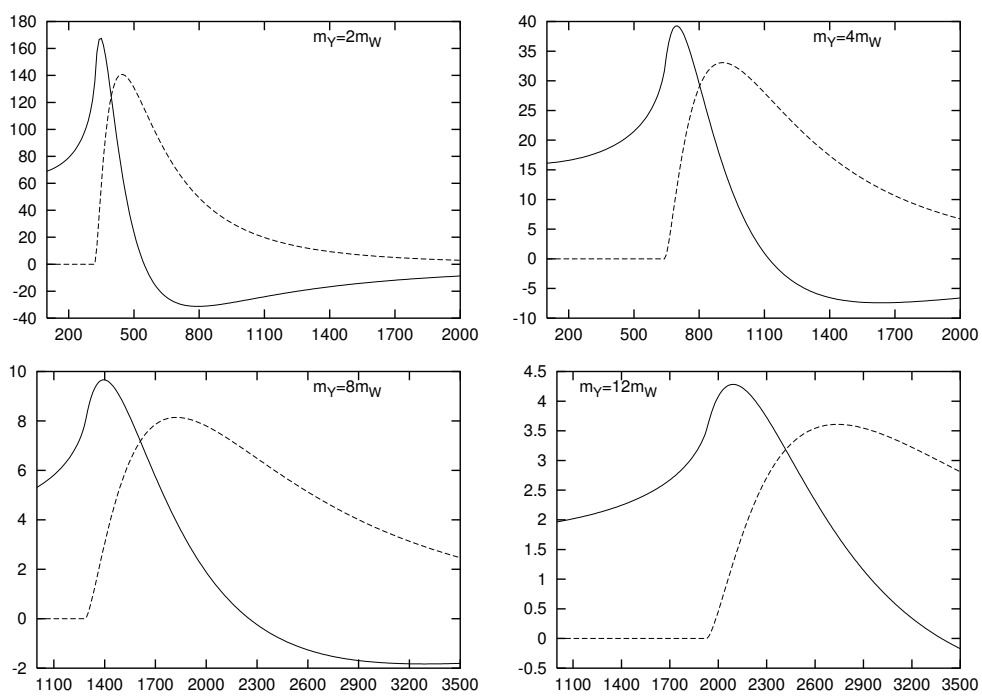

FIG. 6: The same as in Fig. 5 but for the $\Delta Q_{V}$ form factor.

are induced by the following $S U_{L}(2) \times U_{Y}(1)$-invariant dimension-six operators

$$
\begin{aligned}
\mathcal{O}_{W B} & =\frac{\alpha_{W B}}{\Lambda^{2}}\left(\phi^{\dagger} \mathbf{W}_{\mu \nu} B^{\mu \nu} \phi\right), \\
\mathcal{O}_{W} & =\frac{\alpha_{W}}{\Lambda^{2}} \frac{\epsilon_{i j k}}{3 !} W_{\lambda}^{i \mu} W_{\nu}^{j \lambda} W_{\mu}^{k \nu},
\end{aligned}
$$

where $\phi$ is the SM Higgs doublet and $\mathbf{W}_{\mu}=W_{\mu}^{i} \sigma^{i} / 2$, with $\sigma^{i}$ the Pauli matrices. The $\alpha$ constants, which parametrize the details of the underlying physics, could be determined once the fundamental theory is known. In addition, $\Lambda$ is the new physics scale. It turns out that the $\mathcal{O}_{W B}$ and $\mathcal{O}_{W}$ operators induce contributions to $\Delta \kappa_{V}$ and $\Delta Q_{V}$, respectively. Also, it has been shown that these operators can only be induced by the fundamental theory at one-loop or higher orders [49]. Assuming that these operators are induced at the one-loop level in the full theory, the associated $\alpha$ constant must contain a factor of $1 / 16 \pi^{2}$ together with an additional coefficient $g$ or $g^{\prime}$ for each gauge field. Taking 
the bilepton mass as the new physics scale, it is natural to assume

$$
\begin{aligned}
\Delta \kappa_{V} & \sim \frac{g g^{\prime}}{16 \pi^{2}}\left(\frac{m_{W}}{m_{Y}}\right)^{2} f\left(m_{Y}, m_{W}\right), \\
\Delta Q_{V} & \sim \frac{g^{2}}{16 \pi^{2}}\left(\frac{m_{W}}{m_{Y}}\right)^{2} g\left(m_{Y}, m_{W}\right),
\end{aligned}
$$

where $f\left(m_{Y}, m_{W}\right)$ and $g\left(m_{Y}, m_{W}\right)$ stand for the dimensionless loop functions, whose structure depends on the details of the underlying physics. Since the new physics effects are of decoupling nature, the loop functions $f\left(m_{Y}, m_{W}\right)$ and $g\left(m_{Y}, m_{W}\right)$ are expected to be of order $O(1)$ at most. Under this assumption, a straightforward evaluation shows that $\Delta \kappa_{V}$ goes from $2.2 \times 10^{-5}$ to $0.97 \times 10^{-5}$ for $8 m_{W}<m_{Y}<12 m_{W}$, whereas $\Delta Q_{V}$ ranges from $4.1 \times 10^{-5}$ to $1.8 \times 10^{-5}$. This simple qualitative discussion shows that our results are in agrement with those expected in a decoupling scenario of physics beyond the Fermi scale.

\section{CONCLUSIONS}

There is a plenty of good reasons to expect the appearance of new physics beyond the SM, but it still remains a mystery how and where this class of effects would show up. Any new particles would arise by direct production if there is enough energy available, or through their virtual effects on some observable. The last scenario seems to be the most promising if the new particles have masses much larger than the Fermi scale. In this case, high precision measurements are needed in order to detect any deviation from the SM predictions. We have examined this possibility via the radiative corrections to the $W W \gamma$ and $W W Z$ vertices, which would play a special role at NLC experiments. The one-loop contribution to these couplings from the new gauge bosons predicted by the minimal 331 model was studied in a $S U_{L}(2) \times U_{Y}(1)$-invariant way by introducing a nonlinear quantization method. This scheme, even though conventional in the sense that it is based on BRST symmetry, enables one to assess the new physics effects predicted by the 331 model on the SM Green functions through a quantum action that is invariant under the electroweak group. Special emphasis was put on discussing the similarities between our quantization method and the BFM. The main ingredient shared by both methods is that they allow one to construct gauge-invariant quantum actions. It is worth emphasizing however that while the BFM can be used at all energies, as gauge-invariance is preserved with respect to the gauge group of the full theory, the one presented here is only appropriate to study heavy physics effects on low-energy (SM) Green functions. In the latter case the complete quantum action is only invariant under a subgroup of the theory. The $S U_{L}(2) \times U_{Y}(1)$ invariance of the loop amplitudes associated with the $W W \gamma$ and $W W Z$ vertices was showed and special emphasis was put on the inherent simplicity of the calculation. Our results show that for a relative light bilepton, with mass in the range $2 m_{W}<m_{Y}<6 m_{W}$, both $\Delta \kappa_{V}$ and $\Delta Q_{V}$ take values within the range of the SM contribution. On the other hand, for a more heavy bilepton, with mass in the range $8 m_{W}<m_{Y}<12 m_{W}$, the form factors remain essentially uniform and are of the order of $10^{-6}$. It means that they are, respectively, two and one order of magnitude smaller than their SM counterpart. It was also shown that our results are in agreement with

the expectations arising from a decoupling scenario of new physics, as argued in the light of the effective Lagrangian approach. Our results suggest that it would be necessary a high experimental sensitivity in order to detect the virtual effects of new massive gauge bosons because it is hard that any heavy excitations arising from a renormalizable full theory could be much larger than the SM radiative corrections.

\section{Acknowledgments}

We acknowledge support from SNI and Conacyt (México). The work of JMD was supported by Conacyt under grant U44515-F.

[1] W. A. Bardeen, R. Gastmans, and B. Lautrup, Nucl. Phys. B46, 319 (1972).

[2] G. Couture and J. N. Ng, Z. Phys. C 35, 65 (1987); G. Couture, J. N. Ng, J. L. Hewett, and T. G. Rizzo, Phys. Rev. D 36, 859 (1987); G. Tavares-Velasco and J. J. Toscano, Phys. Rev. D 69, 017701 (2004); C. L. Bilchak, R. Gastmans, and A. Van Proeyen, Nucl. Phys. B273, 46 (1986); G. Couture, J. N. Ng, J. L. Hewett, and T. G. Rizzo, Phys. Rev. D 38, 860 (1988); A. B. Lahanas and V. C. Spanos, Phys. Lett. B 334, 378 (1994); T. M. Aliev, ibid. 155, 364 (1985); A. Arhrib, J. L. Kneur, and G. Moultaka, ibid. 376, 127 (1996); N. K. Sharma, P. Saxena, S. Singh, A. K. Nagawat, R. S. Sahu, Phys. Rev. D 56, 4152 (1997). 
[3] G. Tavares-Velasco and J. J. Toscano, Phys. Rev. D 65, 013005 (2002); J. L. García-Luna, G. Tavares-Velasco, and J. J. Toscano, Phys. Rev. D 69, 093005 (2004).

[4] C. Becchi, A. Rouet, and A. Stora, Commun. Math. Phys. 42, 127 (1975); Ann. Phys. 98, 287 (1976); I. V. Tyutin, Lebedev Report No. FIAN 39 (1975) (unpublished).

[5] B. S. DeWitt, Phys. Rev. 162, 1195 (1967); Dynamical Theory of Groups and Fields, Gordon and Breach, New York (1965); C. J. Isham, R. Penrose, and D. W. Sciama, Quantum Gravity 2, Oxford University Press, Oxford (1981); G. t'Hooft, Acta Universitatis Wratislavensis 368, 345 (1976); M. T. Grisaru, P. van Nieuwenhuizen, and C. C. Wu, Phys. Rev. D 12, 3203 (1975); H. Kluberg-Stern and J. B. Zuber, Phys. Rev. D 12, 4823159 (1975); D. G. Boulware, Phys. Rev. D 23, 389 (1981); C. F. Hart, Phys. Rev. D 28, 1993 (1983); L. F. Abbott, Nucl. Phys. B185, 189 (1981); Acta Phys. Pol. B 13, 33 (1982); L. F. Abbott, M. T. Grisaru, and R. K. Schaefer, Nucl. Phys. B229, 372 (1983).

[6] For the BFM formulation of the electroweak theory see: A. Denner, G. Weglein, and S. Dittmaier, Nucl. Phys. B440, 95 (1995); Acta Phys. Polon. B 27, 3645 (1996).

[7] See for instance: S. J. Gates Jr., M. T. Grisaru, M. Rocek, and W. Siegel, Superspace, Frontiers in Physics 58, The Benjamin-Cummings Publishing Company, Advanced Book Program, Reading, Massachusetts (1983) and references therein; S. Ichinose and M. Omote, Nucl. Phys. B203, 221 (1982); D. M. Capper and A. MacLean, Nucl. Phys. B203, 413 (1982); P. S. Howe, G. Papadopoulos and K. S. Stelle, Nucl. Phys. B296, 26 (1988); K. A. Meissner, Acta Phys. Polon. B 17, 409 (1986); J. Antikainen, M. Chaichian, N. R. Pantoja, and J. J. Salazar, Phys. Lett. B 242, 412 (1990); K.-I. Kondo, Int. J. Mod. Phys. A 16, 1303 (2001); C. Becchi and R. Collina, Nucl. Phys. B562, 412 (1999); Qun Wang et al., Int. J. Mod. Phys. E 10, 483 (2001); J. P. Bornsen and A. E. M. van de Ven, Nucl. Phys. B657, 257 (2003); D. J. Toms, Phys. Rev. D 27, 1803 (1983); M. Omote and S. Ichinose, Phys. Rev. D 27, 2341 (1983); S. Mukhi, Nucl. Phys. B264, 640 (1986); A. Rebhan, Z. Phys. C 30, 309 (1986); P. A. Grassi, Nucl. Phys. B462, 524 (1996); Xiao-Yuan Li and Yi Liao, Phys. Lett. B 356, 68 (1995); P. A. Grassi, Nucl. Phys. B560, 499 (1999); A. Denner, G. Weiglein, and S. Dittmaier, Phys. Lett. B 333, 420 (1994); Nucl. Phys. Proc. Suppl. B37, 87 (1994); Nucl. Phys. B440, 95 (1995); Z. Bern and A. G. Morgan, Phys. Rev. D 49, 6155 (1994); L. G. Cabral-Rosetti, J. Bernabeu, J. Vidal, and A. Zepeda, Eur. Phys. J. C 12, $633(2000)$.

[8] J. M. Cornwall, Phys. Rev. D 26, 1453 (1982); J. M. Cornwall and J. Papavassiliou, Phys. Rev. D 40, 3474 (1989); J. Papavassiliou, Phys. Rev. D 41, 3179 (1990).

[9] J. Papavassiliou, Phys. Rev. D 51, 856 (1995).

[10] J. Papavassiliou, Phys. Rev. D 47, 4728 (1993).

[11] J. Papavassiliou, Phys. Rev. D 41, 3179 (1990).

[12] G. Degrassi, B. A. Kniehl, and A. Sirlin, Phys. Rev. D 48, R3963 (1993).

[13] G. Degrassi and A. Sirlin, Phys. Rev.D 46, 3104 (1992).

[14] J. Papavassiliou and K. Philippides, Phys. Rev. D 48, 4255 (1993).

[15] J. Papavassiliou and K. Philippides, Phys. Rev. D 52, 2355 (1995).

[16] A. Denner, G. Weiglein, and S. Dittmaier, Phys. Lett. B 333, 420 (1994); S. Hashimoto, J. Kodaira, Y. Yasui, and K. Sasaki, Phys. Rev. D 50,7066 (1994).

[17] J. Papavassiliou, Phys. Rev. Lett. 84, 2782 (2000); Phys. Rev. D 62, 045006 (2000).

[18] D. Binosi and J. Papavassiliou, Phys. Rev. D 66, 076010 (2002).

[19] I. A. Batalin and G. A. Vilkovisky, Phys. Rev. D 28, 2567 (1983); J. Gomis, J. Paris, and S. Samuel, Phy. Rep. 259, 1 (1995) and references therein.

[20] D. Binosi and J. Papavassiliou, Phys. Rev. D 66, 025024 (2002).

[21] D. Binosi and J. Papavassiliou, Phys. Rev. D 66, R111901 (2002).

[22] D. Binosi and J. Papavassiliou, J. Phys. G 30, 203 (2004); D. Binosi, J. Phys. G 30, 1021 (2004).

[23] K. Fujikawa, Phys. Rev. D 7, 393 (1973).

[24] The most general gauge-fixing functions of $R_{\xi}$ type, nonlinear in both the vector and scalar sectors, were introduced in U. Cotti, J. L. Díaz-Cruz, and J. J. Toscano, Phys. Lett. B 404, 308 (1997) and their implications on the ghost sector discussed with detail in J. G. Méndez and J. J. Toscano, Rev. Mex. Fís. 50, 346 (2004).

[25] L. D. Faddeev and V. N. Popov, Phys. Lett. B 25, 29 (1967).

[26] M. Bace and N. D. Hari Dass, Ann. Phys. 94, 349 (1975); M. B. Gavela, G. Girardi, C. Malleville, and P. Sorba, Nucl. Phys. B193, 257 (1981); N. M. Monyonko, J. H. Reid, and A. Sen, Phys. Lett. B 136, 265 (1984); N. M. Monyonko and J. H. Reid, Phys. Rev. D 32, 962 (1985); G. Jikia and A. Tkabladze, Phys. Lett. B 323, 453 (1994); ibid. 332, 441 (1994); G. Jikia, Phys. Lett. B 298, 224 (1993); Nucl. Phys. B405, 24 (1993); ibid. 412, 57 (1994); D. A. Dicus and C Kao, Phys. Rev. D 49, 1265 (1994); A. Abbasabadi, D. Bowser-Chao, D. A. Dicus, and W. W. Repko, Phys. Rev. D 52, 3919 (1995); J. M. Hernández, M. A. Pérez, G. Tavares-Velasco, and J. J. Toscano, Phys. Rev. D 60, 013004 (1999); U. Cotti, J. L. Díaz-Cruz and J. J. Toscano, Phys. Rev. D 62, 035009 (2000); J. L. Díaz-Cruz, J. Hernández-Sánchez, and J. J. Toscano, Phys. Lett. B 512, 339 (2001); J. Hernández-Sánchez, M. A. Pérez, G. Tavares-Velasco, and J. J. Toscano, Phys. Rev. D 69, 095008 (2004).

[27] H. Min, T. Lee, and P. Y. Pac, Phys. Rev. D 32, 440 (1985); H. Hata and I. Niigata, Nucl Phys. B 389, 133 (1993); K.-I. Kondo, Phys. Rev. D 58, 105019 (1998); T. Shinohara, T. Imai, and K.-I. Kondo, Int. J. Mod. Phys. A 18, 5733 (2003).

[28] F. Pisano and V. Pleitez Phys. Rev. D 46, 410 (1992).

[29] P. H. Frampton, Phys. Rev. Lett. 69, 2889 (1992).

[30] For some of the most recent works on the phenomenology and some theoretical topics of 331 models see: Alex G. Dias, C. A. de S. Pires, V. Pleitez, and P. S. Rodrigues da Silva, arXiv: hep-ph/0503192 Alex G. Dias, A. Doff, C. A. de S. Pires, and P. S. Rodrigues da Silva, arXiv: hep-ph/0503014 G. Tavares-Velasco and J. J. Toscano, Phys. Rev. D 70, 
053006 (2004); Alex G. Dias, Phys. Rev. D 71, 015009 (2005); J. Alexis Rodríguez and M. Sher, Phys. Rev. D 70, 117702 (2004); Alex G. Dias, R. Martínez, and V. Pleitez, Eur. Phys. J. C 39, 101 (2005); J. C. Montero, V. Pleitez, and M. C. Rodriguez, Phys. Rev. D 70, 075004 (2004).

[31] M. A. Pérez, G. Tavares-Velasco, and J. J. Toscano, Phys. Rev. D 69, 115004 (2004).

[32] D. Ng, Phys. Rev. D 49, 4805 (1994); J. T. Liu and D. Ng, Z. Phys. C 62, 693 (1994).

[33] C. P. W. Group et al., arXiv: hep-ph/0412251 and references therein.

[34] K. J. F. Gaemers and G. J. Gounaris, Z. Phys. C 1, 259 (1979); K. Hagiwara, R. D. Peccei, D. Zeppenfeld, and K. Hikasa, Nucl. Phys. B282, 253 (1987); U. Baur and D. Zeppenfeld, ibid. B308, 127 (1988); B325, 253 (1989).

[35] M. Böhm et al. Nucl. Phys. B304, 463 (1988); C.-rim Ahn, M. E. Peskin, B. W. Lynn, and S. B. Selipsky, ibid. B309, 221 (1988); J. Fleischer, F. Jegerlehner, and M. Zralek, Z. Phys. C 42, 409 (1989); J. Fleischer, K. Kolodziej, and F. Jegerlehner, Phys. Rev. D 47, 830 (1993); W. Beenakker et al. Nucl. Phys. B410, 245 (1993); W. Beenakker, A. Denner, S. Dittmaier, and R. Mertig, Phys. Lett. B 317, 622 (1993).

[36] A. Denner and T. Sack, Z. Phys. C 45, 439 (1990); D. Y. Bardin, W. Beenakker, and A. Denner, Phys. Lett. B 317, 213 (1993); R. G. Stuart, ibid. 262, 113 (1991); A. Aeppli, G. J. van Oldenborgh, and D. Wyler, Nucl. Phys. B428, 126 (1994); W. Beenakker and A. Denner, Int. J. Mod. Phys. A 9, 4837 (1994); K. Melnikov and O. Yakovlev, Nucl. Phys. B471, 90 (1996); W. Beenakker, A. P. Chapovsky, and F. A. Berends, Phys. Lett. B 411, 203 (1997); A. Denner, S. Dittmaier, and M. Roth, Nucl. Phys. B519, 39 (1998); Phys. Lett. B 429, 145 (1998); W. Beenakker, F. A. Berends, and A. P. Chapovsky, Nucl. Phys. B548, 3 (1999); A. Denner, S. Dittmaier, M. Roth, and D. Wackeroth, ibid. B 560, 33 (1999); Phys. Lett. B 475, 127 (2000).

[37] J. Ellison and J. Wudka, Ann. Rev. Nucl. Part. Sci. 48, 33 (1998).

[38] B. Holdom, Phys. Lett. B 258, 156 (1991).

[39] S. Alam, Phys. Rev. D 50, 124 (1994); 50, 148 (1994); 50, 174 (1994); S. Alam, K. Hagiwara, S. Kanemura, R. Szalapski, and Y. Umeda, Phys. Rev. D 62, 095011 (2000); T. Hahn, Nucl. Phys. B609, 344 (2001); K. Hagiwara, S. Kanemura, M. Klasen, and Y. Umeda, Phys. Rev. D 68, 113011 (2003).

[40] E. N. Argyres et al., Nucl. Phys. B391, 23 (1993).

[41] J. Papavassiliou and K. Philippides, Phys. Rev. D 48, 4255 (1993).

[42] J. Alitti et al., Phys. Lett. B 277, 194 (1992); K. Ackerstaff et al., Phys. Lett. B 397, 147 (1997); K. Ackerstaff et al., Eur. Phys. J. C 2, 597 (1998); P. Abreu et al., Phys. Lett. B 397, 158 (1997); M. Acciarri et al., Phys. Lett. B 398, 223 (1997); 403, 168 (1997); 413, 176 (1997); R. Barate et al., ibid. 422, 369 (1998).

[43] F. Abe et al., Phys. Rev. Lett. 74, 1936 (1995); 75, 1017 (1995); 78, 4536 (1997); S. Abachi et al., ibid. 75, 1034 (1995); 78, 3634 (1997); 75, 1023 (1995); 77, 3303 (1996); B. Abbott et al., ibid. 79, 1441 (1997); S. Abachi et al., Phys. Rev. D 56, $6742(1997)$.

[44] A. Arhrib, J. L. Kneur, and G. Moultaka, Phys. Lett. B 376, 127 (1996); E. N. Argyres, A. B.Lahanas, C. G. Papadopoulos, and V. C. Spanos, ibid. 383, 63 (1996).

[45] L. Willmann et al., Phys. Rev. Lett. 82, 49 (1999).

[46] V. Pleitez, Phys. Rev. D 61, 057903 (2000); P. Das and P. Jain, ibid. 62, 075001 (2000); P. H. Frampton and A. Rasin, Phys. Lett. B 482, 129 (2000).

[47] M. B. Tully and G. C. Joshi, Phys. Lett. B 466, 333 (1999).

[48] M. B. Tully and G. C. Joshi, Int. J. Mod. Phys. A 13, 5593 (1998).

[49] C. Arzt, M. B. Einhorn, and J. Wudka, Nucl. Phys. B433, 41 (1995). 